\title{
Keratoderma as a Paraneoplastic Sign on NSCLC Patient - Case Report
}

Esti Shamba ${ }^{1^{*}}$, Ephraim Shamba ${ }^{2}$ and Nir Peled ${ }^{3}$

${ }^{1}$ Research Coordinator, Institute of Pulmonology, Schneider Children's Medical Center of Israel, Israel

${ }^{2}$ Family physician, Clalit Health Services, Jerusalem, Israel

${ }^{3} \mathrm{Head}$, Institute of Oncology, Soroka Medical Center, Ben-Gurion University, Beer Sheva, Israel

*Corresponding author: Shamba E, Research Coordinator, Institute of Pulmonology, Schneider Children's Medical Center of Israel, Israel, Tel: +972-523263571; Email: stshamba@gmail.com

Received date: March 15, 2018; Accepted date: May 08, 2018; Published date: May 15, 2018

Copyright: $@ 2018$ Shamba E. This is an open-access article distributed under the terms of the Creative Commons Attribution License, which permits unrestricted use, distribution, and reproduction in any medium, provided the original author and source are credited.

\begin{abstract}
Palmoplantar keratoderma is a dermatosis that presents as hyperkeratosis of the palms and soles. Skin disorders of the feet can affect the glabrous skin on the dorsal aspects, or the thick skin on the plantar aspectsthereof, or both. Some can affect one foot, and others both. These diseases can be inflammatory, genetically inherited, infectious orneoplastic in origin. It is important to identify them and to start treatment early. If not treated, some may lead to severe pain on walking, inability to wear shoes, or even amputation with serious disability.

We present a patient with severe treatment-resistant keratoderma of the feet, in addition to a long-standing dry cough. Because of the cough, a CT scan of the chest was done and a $2.5 \times 2.5 \mathrm{~cm}$ nodule was detected in the left lower lobe of the lung. A biopsy confirmed the diagnosis of non-small cell lung cancer (NSCLC) stage IA. It was resected entirely with left lower lobectomy. The keratoderma resolved spontaneously within a few weeks of surgery.
\end{abstract}

Keywords: NSCLC; Keratoderma; Hyperkeratosis

\section{Introduction}

Palmoplantar Keratoderma (PPK) is a dermatosis that presents with hyperkeratosis. This marked thickening of the skin of the palms and soles can be a paraneoplastic syndrome pointing to a relation between an internal malignancy [1] and a cutaneous disorder, even at a remote location.

Palmoplantar keratoderma can also be associated with visceral malignancies, e.g. esophageal, gastric, pulmonary, and urinary/bladder carcinomas [2]. Paraneoplastic syndromes associated with lung cancer include neurologic, endocrine, dermatologic, rheumatologic, hematologic, and ophthalmologic syndromes, as well as glomerulopathy and coagulopathy [3].

In 1868, Hebra was the first to suggest that skin pigmentation could indicate the presence of visceral cancer [4]. Since then, only 49 cases have appeared in the literature, 41 of which (84\%) were associated with a neoplasm, most commonly of the lung. Several patients also had pruritus, palmoplantar keratoderma, ichthyosis, vesiculobullous lesions, and/or eosinophilia. Histopathologic findings are nonspecific. The skin findings usually disappear with therapy for the underlying malignancy [5].

\section{Case Report}

A 71-year-old male non-smoker, with a history of repair of Tetralogy of Fallot (TOF) at the age of 31, colectomy for colon cancer (T1NOMO) at 59, and complete cure of hepatitis $\mathrm{C}$ at 67 , presented in January 2017 with keratoderma on the soles of the feet and dermatitis accompanied by an itchy rash over the entire body. Local skin treatments were ineffective.
The patient was hospitalized withxerotic skin and deep linear cracks on both feet. Fungus and scabies tests were negative. Over 4 months he was treated with local softening agents such asurea - lactic acid CD cream, anti-pruritus agents likebutenafine hydrochloride and antiseptic solutions like povidone iodine. Local steroid preparations such as clobetasol propionate also had no effect. Even Phototherapy (PUVA) was tried without benefit.

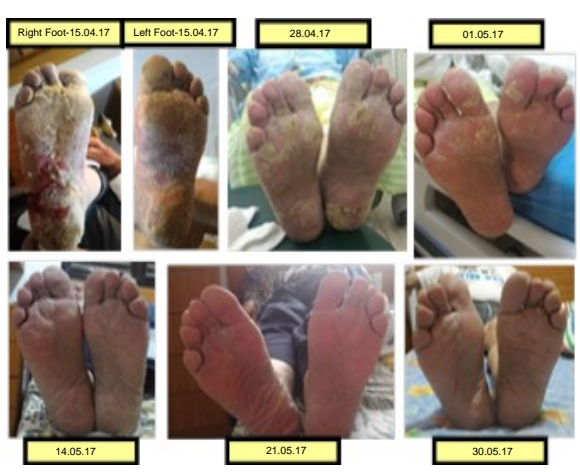

Figure 1: Change in the severity of the Keratoderma after surgery to remove the lesion from the lung.

Right foot on 15.04.17 before surgery.

Left foot on 15.04 .17 before surgery.

8 days post-surgery.

10 days post-surgery.

24 days post-surgery.

31 days post-surgery.

40 days post-surgery. 
In light of a longstanding dry cough, a chest CT was performed, which indicated a coin lesion, measuring $2.5 \times 2.5 \mathrm{~cm}$, in the lower left lobe of the lung. A biopsy confirmed the diagnosis of non-small cell lung cancer (NSCLC). Following otherwise normal PET CT, the patient underwent left lower lobectomy and was staged pT2N0M0. The pathology report was consistent with a moderately differentiated adenocarcinoma, with predominant lepidic growth. Rapid improvement of the keratoderma was observed within days of the removal of the tumor. Figure 1 illustrates the gradual resolution, over a period of seven weeks (15/04/2017 - 30/05/2017).

\section{Discussion}

Cutaneous paraneoplastic syndromes are a large group of dermatoses that may be associated with visceral malignancies. A broad spectrum of paraneoplastic dermatoses is associated with lung cancer [6]. This case report demonstrates that keratoderma of the feet and hands may indicate the presence of lung cancer and this phenomenon can be reversible on removal of cause.

This case demonstrates the importance of the primary physician's awareness of neoplastic phenomena with certain medical conditions, especially when the primary etiology is elusive, or when the condition is not responsive to standard treatment.

As noted above paraneoplastic dermatoses are rare dermatologic entities that are difficult to diagnose [7]. Therefore recognition of external clues is important to facilitate both early diagnosis and prompt treatment of the internal disorder. Early recognition is especially valuable in a patient with an internal malignant disease because intervention may significantly affect survival [8].

\section{References}

1. Thiers BH, Sahn RE, Callen JP (2009) Cutaneous manifestations of internal malignancy. CA: ACancer J Clinicians59:73-98.

2. Kallini JR, Sadeghani K, Khachemoune A (2017) Paraneoplastic palmoplantar keratoderma secondary to metastatic uterine adenocarcinoma. Cutis 99: E32-5.

3. Kanaji N, Watanabe N, Kita N, Bandoh S, Tadokoro A, et al. (2014) Paraneoplastic syndromes associated with lung cancer. World J Clin Onco 5:197.

4. Sneddon IB. (1970) Cutaneous manifestations of visceral malignancy. Postgraduate Med J 46: 678.

5. Boyd AS, Neldner KH, Menter A (1992) Erythema gyratum repens: a paraneoplastic eruption. J American Acad Derma 26: 757-762.

6. Youssef M, Kechida M, Cheikhmohamed S, Moussa A (2014) Multiple paraneoplastic syndromes revealing non-small cell lung carcinoma. The Pan African Med J19.

7. Ortega-Loayza AG, Ramos W, Gutierrez EL, Paz PC, Bobbio L, et al. (2010) Cutaneous manifestations of internal malignancies in a tertiary health care hospital of a developing country. Anais brasileiros de dermatologia 85: 736-742.

8. Poole S, Fenske NA (1993) Cutaneous markers of internal malignancy. I. Malignant involvement of the skin and the genodermatoses. J American Acad Derma 28: 1-3. 